\title{
LITERATURE REVIEW \\ Difficulties in the treatment of recurrent juvenile laryngeal papillomatosis associated with pemphigus vulgaris
}

\author{
Codrut Sarafoleanu',2, Gheorghe Lapicus', Ana Isabela Lupu ${ }^{3}$, Vlad Budu ${ }^{2,4}$ \\ ${ }^{1}$ ENT\&HNS Department, "Sfanta Maria” Hospital, Bucharest, Romania \\ 2"Carol Davila" University of Medicine and Pharmacy, Bucharest, Romania \\ ${ }^{3}$ Pediatric Hospital, Ploiesti, Romania \\ 4"Prof. Dr. D. Hociota" Institute of Phono-Audiology and Functional Surgery, Bucharest, Romania
}

\begin{abstract}
Recurrent laryngeal papillomatosis is a disease caused by human papillomaviruses (HPVs), which currently does not benefit from a curative treatment. Due to the fact that HPV has the action of modifying cellular DNA, with changes in the expression of interleukins and interferon, with insufficient maturation of T cells and intracellular overpopulation of immunosuppressive cells, the association of RRP (Recurrent Respiratory Papillomatosis) with an autoimmune disease may cause particular difficulties in the therapeutic management of patients diagnosed with RRP.

Immunosuppressive medication negatively influences the development of papillomatosis, increasing the number of local relapses and, respectively, the need for surgical intervention due to the increased viral multiplication and the proliferation of papillomatous lesions. In order to exemplify the difficulties encountered in treating RRP associated with an autoimmune disorder, the authors present the case of a 21-year-old patient diagnosed with juvenile recurrent laryngeal papillomatosis genotype 6 , with multiple antecedent surgeries, who was diagnosed with pemphigus vulgaris at the age of 19 . The peculiarity of the case lies in the difficulty of managing the RRP associated with an immunosuppressive disorder whose therapeutic indication is cortisone and immunosuppressive treatment, which led to exacerbation of viral multiplication and proliferation of papillomatous lesions.
\end{abstract}

KEYWORDS: recurrent respiratory papillomatosis, cidofovir, human papillomavirus, pemphigus vulgaris, immunosuppressive disorder.

\section{INTRODUCTION}

Recurrent respiratory papillomatosis is a disease caused by human papillomaviruses (HPVs), characterized by the development of papillomas at the level of the aero-digestive tract. Papillomas are virulent excrescences, that are located especially in the larynx, and involve HPV genotypes $6,11,30,16,18$; the rest are distributed in the nasopharynx, oropharynx, lungs, trachea and bronchi with the involvement of HPV 2, 13, 32, 40, 57, 16, $18^{1-3}$.

Recurrent respiratory papillomatosis (RRP) is a benign disease, that tends to recur and spread to the lower respiratory tract. The evolution of the disease is variable, with spontaneous remissions or aggressive growths of papillomatous tumors and sometimes with their malignant transformation.

\section{CHARACTERISTICS OF HUMAN PAPILLOMAVIRUS}

Papillomaviruses belong to the Papillomavirus genus of the Papovaviridae family. They are non-enveloped viruses, with a diameter between $50-55 \mathrm{~nm}$, with the icosahedral capsule composed of 72 capsomers, containing a circular double-stranded DNA genome made up of 7900 nucleotide pairs ${ }^{4}$. HPV can cause lytic infection in permissive cells producing infectious virions and persistent infection in non-permissive cells that can lead to malignant transformation ${ }^{4}$.

There are more than 100 genotypes of human papillomaviruses (HPVs), which can infect mucous membranes or skin epithelium ${ }^{5}$. Viruses have the property of causing benign proliferative lesions within the epi- 
thelial tissues they infect. HPVs are classified as low- or high-risk genotypes, reflecting the risk of malignant transformation ${ }^{6}$. The high oncogenic risk of HPV genotypes is well known, being considered as etiological agents of the majority of cervical cancers, of some types of anogenital cancers, as well as some cancers of the head and neck. The most commonly encountered in these types of cancer is HPV 16 subtype $^{7}$.

The current classification system divides HPV strains into subtypes: "low risk" $(6,11,13,40,42-44,54,61,62$, $70,72,74,81)$ and "high risk" (16, 18, 31, 33, 35, 39, $45,51,52,56,58,59,68,73,82)$, depending on their ability to induce neoplasms ${ }^{8,9}$. HPV subtypes 26,53 and 66 can be considered as potentially carcinogenic, due to the extremely small number of infected patients ${ }^{9}$. In benign or low-risk HPV lesions, such as those typically associated with types 6 and $11 \mathrm{HPV}$, the HPV genome exists as circular episomal DNA separated from the host cell nucleus. In malignant lesions, the genome of subtypes HPV 16 and 18 is usually integrated into the DNA of the host cells ${ }^{10-12}$. The integration of the viral genome into the host cell genome is considered a distinctive sign of malignant transformation.

Although it has never been directly demonstrated, it is assumed that the onset of HPV infection is initiated in the basal compartment of the host epithelium $^{13}$. The specific mechanism used by HPV to penetrate basal layer cells is unclear.

Once the HPV particles enter the host cell and translocate their DNA to the nucleus, they must initially be based on the replicative cellular mechanisms of the host genome, because HPV encodes only a component of the equipment required to initiate its own DNA replication ${ }^{10,11,13,14}$. This is why HPV is supposed to initiate their infectious viral cell cycle in the cells within the basal compartment, because they are the only cells in the epithelium that have active proliferative mechanisms.

A particular feature of HPV infections is the persistence of the infection for several years. In cases of high-risk HPV strains, this persistence is a precondition for the development of neoplasms associated with these viruses ${ }^{13}$. In order for the virus to persist for a long time, it must maintain its genome in the basal compartment where multiple cell divisions occur. Moreover, the virus must produce an amplification of cells within the basal compartment that hosts the viral genome. A possible explanation, both for the persistence and extension of the infected cells, is that the virus infects epithelial cells with stem cell properties ${ }^{15-17}$. The group of cells populating the local area with HPV-positive cells are derived and amplified from these stem cells. This is an attractive hypothesis, although these types of cells are rare.

An alternative explanation is the fact that HPV would temporarily amplify non-stem cells and extend their lifespan, which leads to both viral persistence and expansion of infected cells to their original site of infection. Perhaps this alternative explanation reflects the fact that high-risk HPV strains have the ability to cause epithelial cell neoplasms ${ }^{13,17}$.

\section{PATHOGENESIS OF RECURRENT RESPIRATORY PAPILLOMATOSIS}

It is not known exactly why only a very small fraction of individuals exposed to HPV develop recurrent respiratory papillomatosis (RRP). An increased frequency of HLA-DRB $1 * 0102$ was observed in Caucasian patients with RRP, suggesting that this allele predisposes individuals to $\mathrm{RRP}^{2,3,18}$. Furthermore, alleles HLADRB1*0301, DQB1*0201 and DQB1*0202 are more common in Caucasians with severe disease ${ }^{18,19}$. Instead, the complex HLA-DQB1*0602 correlates with a benign evolution of the disease in white individuals. Alleles DQB1*0201 as well as DQB1*0202 are present, while DQB1*0602 are missing in African-American patients. Interestingly, HLA-DRB $1 * 0301$ and DQB $1 * 0201$ correlated with reduced interferon-gamma expression in patients with $R R P^{18,19}$.

RRP occurs more frequently in healthy patients or with impaired immune status, such as patients with HIV, with organ transplants, who receive immunosuppressive drugs or with congenital immune deficiencies. The systemic immune system functions and determines a systemic response through antibodies following interaction with viral proteins.

The defence against viral infections is mediated by T helper 1 cells (Th1) and T helper 2 (Th2), and involves the interaction between Th1 cells and antigen presenting cells (APC), which produce interleukin (IL) 2 and T-killer or CD8 (CTL) ${ }^{13,19}$. Snowden and colleagues identified and measured by ELISA serum levels of IL-2 and IL-2 receptor ${ }^{20}$.

One mechanism of CTL inhibition in papillomatosis is poor inadequate expression of IL-2 / IFN-y and increased expression of IL-4 / IL-10, which maintains insufficient maturation of CD8+T cells ${ }^{13,19,21}$. Cytokine barriers prevent CTL entry into the cells and there is intracellular overpopulation of FoxP3+ immunosuppressive T cells ${ }^{22,23}$.

Another suppressive hypothesis in RRP involves the mediators of the programmed cell death-1 (PD-1) and its programmed cell death ligand 1 (PD-L1) ${ }^{22,23}$. PD-1 is a receptor for $\mathrm{T}$ cells and is a negative feedback marker for their functioning and indicates CD8+ T cells depletion. The concentration of PD-L1 and CD4+ is elevated in the epithelium of patients with RRP, using immunohistochemical tests.

In HPV infection, dysfunctions of cellular apoptosis were observed. Also, in RRP, increased levels of VEGF (vas- 
cular endothelial growth factor) were recorded ${ }^{13}$.

All these investigated factors will become the target of modern immunological and gene therapies in RRP.

Papillomaviruses exhibit tropism for tegumental squamous epithelial tissue and mucous membranes. At this level, the virus replicates and induces tissue proliferation, generating tumor formations.

Infection is achieved by direct contact (including sexual contact), at the level of even minimal continuity solutions.

Viral replication and gene expression are dependent on the degree of differentiation of the infected epithelial cell, generating persistent infections in the basal layer and active infections in keratinocytes. The infection is usually localized and has a spontaneously regressive evolution.

In certain papillomaviruses, the viral genome may persist intracellularly (through the integration of viral DNA into the host genome), causing recurrences. Certain types cause dysplasia with malignant potential (in combination with carcinogenic cofactors).

Schematically, the evolution of a papillomavirus infection can be reproduced as follows:

1. Epithelial cell infection (tegument, mucous membrane)

2. Local multiplication

3. Tumor mass (palmar, plantar skin, pharyngo-laryngeal mucosa)

4. a. Regression of tumor mass (with virus persistence - latent infection)

or

4. b. Malignant transformation.

High-risk subtypes, especially HPV-16, are etiologically associated with approximately $25 \%$ of head and neck squamous cell carcinomas (HNSCC), especially with those of the oropharynx with which the etiological correlation is greater than $50 \%{ }^{24}$.

\section{CLINICAL AND PARACLINICAL CHARACTERISTICS OF RRP}

Recurrent respiratory papillomatosis is a condition that does not currently benefit from any complete curative treatment. The recurrent nature and the very serious complications it causes involves frequent hospitalizations and very high costs for the health system.

The clinical picture in patients with laryngeal papillomatosis is characterized by dysphonia of varying degrees, dyspnea that can go up to acute respiratory failure due to an obstructive cause. Other less specific symptoms are: cough, recurrent pneumonia, insufficient growth, dysphagia or acute respiratory failure especially in children with acute respiratory infections. RRP may initially be confused with other disorders such as asthma, various allergies, croup,

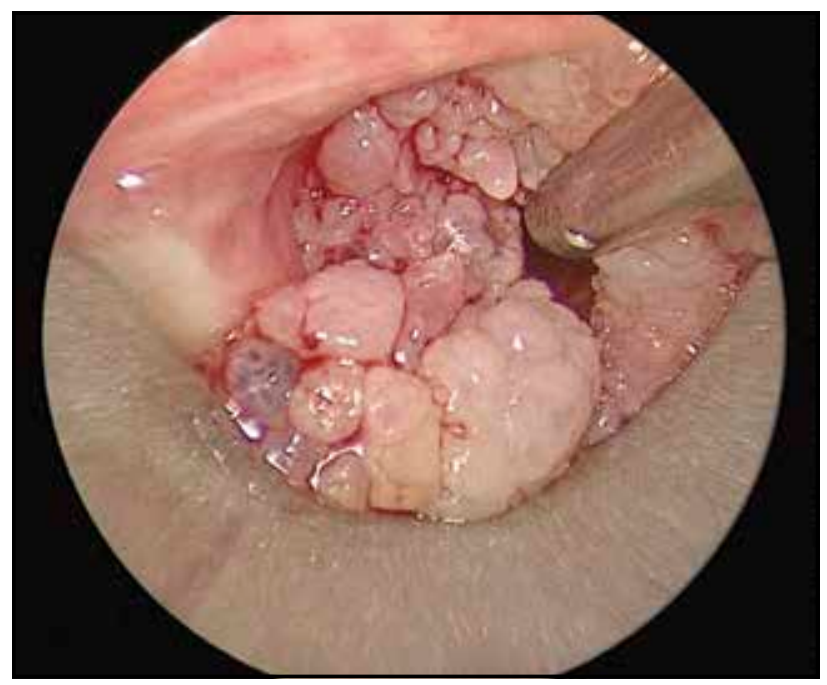

Figure 1. Suspension microlaryngoscopy - laryngeal papillomatosis.

bronchitis, vocal nodules, recurrent pneumonia, developmental delays in children, dysphagia ${ }^{1}$.

The diagnosis of laryngeal papillomatosis is based on detailed anamnesis and clinical examination. The laryngofibroscopic examination helps us to examine in more detail the papillomatous lesions, to assess the origin and extent of the lesions. Suspension microlaryngoscopy with biopsy sampling of the lesions followed by histopathological examination establishes the diagnosis of certainty ${ }^{1-3}$ (Figure 1).

In general, the diagnosis of RRP is a clinical one, based on the direct visualization of the lesions in the nasal vestibule, oral cavity, oropharynx, that will subsequently be confirmed by histopathological examination. Patient investigation should include: indirect laryngoscopy, laryngofibroscopic examination, suspension microlaryngoscopy, biopsy with histopathological examination, genotyping PCR.

It is very important to stage the patient with RRP using the Derkay severity score ${ }^{25}$. It is obtained by summing up the clinical score and the anatomical score (laryngoscopic) and has a value between 0 and 86 points.

Recurrent laryngeal papillomatosis characteristics are as follows: relapse, extension (to the trachea, bronchi and lungs or remote extensions), malignant transformation. In the case of malignant transformation, the most common sites of neoplasia are the larynx or lung, and the histological form is squamous cell carcinoma with keratinizing, non-keratinizing or warty variants. Lesions with moderate or severe dysplasia and keratinization abnormalities (hyperkeratosis, ortho- and parakeratosis) are associated with increased risk of malignant transformation.

There are studies that have found the association between HPV and autoimmune diseases; in this case, HPV may act as a possible cofactor with an increased 
morbidity potential ${ }^{26-29}$. Autoimmune diseases, in general, affect about 5-10\% of the world's developed population ${ }^{30,31}$. The etiology and pathogenesis of autoimmune diseases involve a combination of genetic, immune, infectious, hormonal and environmental factors. Lichen planus, mucous membrane pemphigoid, pemphigus vulgaris and epidermolysis bullosa acquisita are the most common oral and cutaneous autoimmune diseases. They are characterized by chronic lesions that cannot be cured. Human papillomavirus (HPV) is a DNA virus that infects the squamous epithelium of the skin and mucous membranes ${ }^{17}$.

Due to the fact that HPV has the action of modifying cellular DNA, with changes in the expression of interleukins and interferon, with insufficient maturation of $\mathrm{T}$ cells and intracellular overpopulation of immunosuppressive cells, there is a significant decrease in the cell-mediated immune status. As a result, the association of RRP with an autoimmune disease may cause particular difficulties in the therapeutic management of patients diagnosed with RRP.

\section{DIFFICULTIES IN THE TREATMENT OF RRP ASSOCIATED WITH AUTOIMUNE DISEASES}

Although many studies have been conducted regarding the treatment of RRP, the varied results do not indicate any therapeutic variant capable of completely curing the viral infection. The current therapeutic principle is based on surgical and drug treatment ${ }^{1-3}$.

Surgical treatment may involve the use of cold instruments, CO2 LASER vaporization of papillomatous formations, angiolytic LASER treatment or microdebrider ablation.

Regarding adjuvant treatment, several variants are described in the literature, such as: a-interferon, Cidofovir, indole-3-carbinol or bevacizumab - monoclonal antibodies ${ }^{2,8,24,26}$.

The association of an autoimmune disease with laryngeal papillomatosis may be a factor with a negative evolutionary prognosis for the latter, mainly in terms of treatment required in autoimmune disorders.

In autoimmune diseases, the purpose of the treatment is not a curative one, but is mainly aimed at reducing symptoms. The purpose of pharmacological therapy is primarily that of reducing the inflammatory response and the production of autoantibodies. The treatment has two principles - a general (basic) one and a local one.

The general treatment for autoimmune disorders can be symptomatic or immunosuppressive and can act on pro-inflammatory factors (TNF-alpha, IFN-gamma, IL6, Il17, I110) or cell families involved in the biomolecular process of autoimmune diseases (T cells - T4, Ts, Treg -, dendritic cells, plasma cells, endothelial cells) ${ }^{32,33}$.

Immunosuppressive treatment should be consid- ered at the onset of the disease, in combination with corticosteroids. The corticosteroid therapy generally uses prednisone in the attack dose of $1-2 \mathrm{mg} / \mathrm{kg} /$ day until the cessation of new lesions and the obvious improvement of the existing ones, then the dose is reduced until reaching a maintenance dose which keeps the disorder under control ${ }^{34}$.

Steroids should be combined with an adjuvant immunosuppressant, such as azathioprine $(2-3 \mathrm{mg} / \mathrm{kg} \text { daily })^{35}$, mycophenolate mofetil (1 to $3 \mathrm{~g}$ per day) ${ }^{36}$ or methotrexate with doses ranging from 7.5 to $25 \mathrm{mg} /$ week $^{32}$.

The main problem in patients with RRP and autoimmune diseases is that some of the adjuvant immunosuppressive therapies may increase the risk (already produced by systemic steroids) of worsening / recurrence of HPV infection (laryngeal papillomatosis). For this reason, the treatment with azathioprine or mycophenolate mofetil / mycophenolic acid should be avoided in patients with RRP.

To highlight the difficulties encountered in treating RRP in patients with autoimmune disorders, we present the case of a 21-year-old male patient with 2 different medical problems: laryngeal papillomatosis (LP) diagnosed at 10 months, which required numerous ENT surgeries, and pemphigus vulgaris (PV) that started in 2017 and manifested as erosions in the oral cavity, pharynx and larynx. Pemphigus is a bullous dermatosis of autoimmune cause, whose main clinical sign is the formation of intraepithelial bubbles on the skin and mucous membranes. Pemphigus is characterized by acantholysis and immunoglobulin deposits in the space between keratocytes ${ }^{37}$. Patients with pemphigus develop an autoimmune response directed against desmosomes ${ }^{37,38}$. It affects the skin and mucous membranes, being equally met in both sexes with the onset recorded around the age of 40-60 years. The disease is mediated by circulating autoantibodies directed against the surface of keratinocytes. The predisposition to pemphigus is related to genetic factors. Some class II major histocompatibility molecules (MHC), especially alleles of human leukocyte antigen DR4 (DRB1*0402) and human leukocyte antigen DRw6 (DQB1*0503), are common in patients with pemphigus vulgaris ${ }^{39}$.

The patient was treated with oral prednisolone at an initial dose of $75 \mathrm{mg} / \mathrm{d}$ (we suppose this may correspond to $1 \mathrm{mg} / \mathrm{kg}$ ), which was subsequently reduced to $15 \mathrm{mg} /$ day. He also received sulfasalazine, but it was discontinued due to side effects. His pemphigus vulgaris was particularly improved (complete wound healing) and there was a significant decrease in circulating antidesmoglein 3 antibodies. As a complication of PV treatment with systemic steroids, LP relapsed several times and required more frequent surgical procedures. The patient received additional treatment with intralesional cidofovir and quadrivalent HPV vaccine (Gardasil).

At the time of admission to our clinic, the patient had inspiratory dyspnea, dysphonia and moderate 

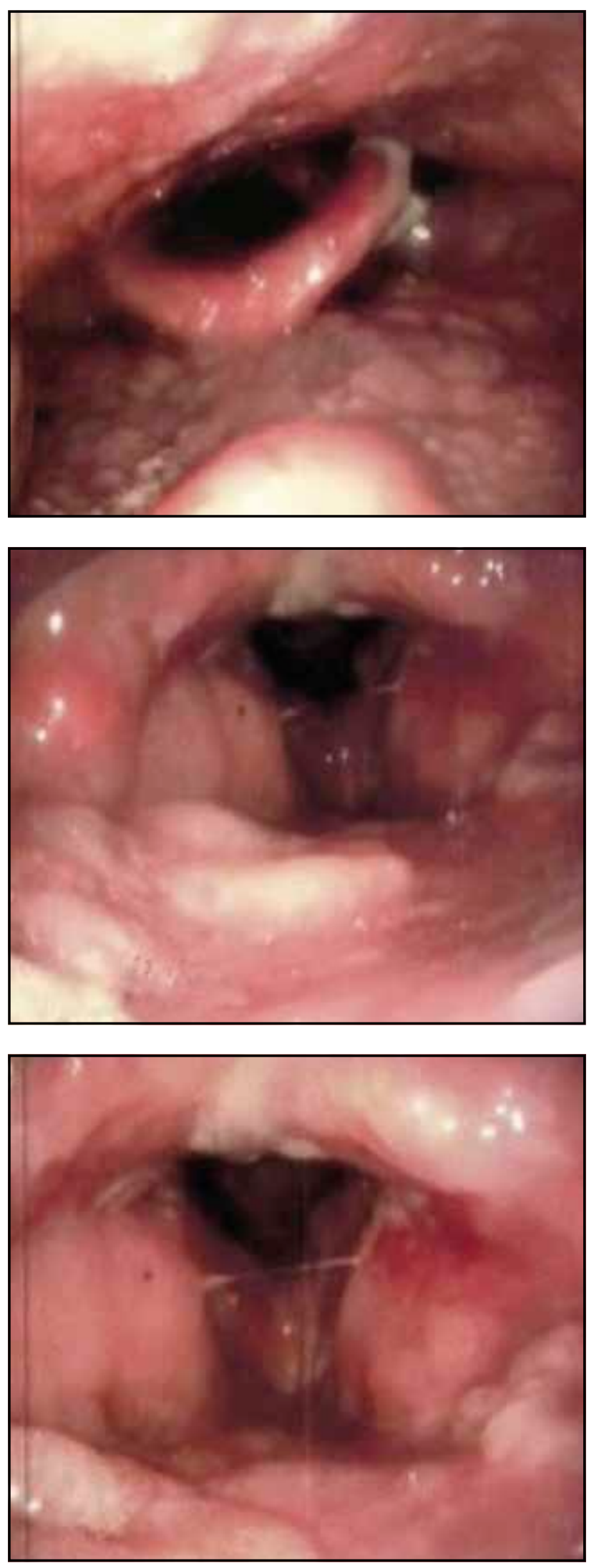

Figure 2. Laryngeal fibroscopy: bullous lesions and erosions on the surface of the pharyngeal-laryngeal mucosa and papillomatous lesions at the level of the vocal cords. dysphagia. The patient was diagnosed at 10 months with recurrent juvenile laryngeal papillomatosis (genotype 6) - for which over time he was operated 87 times both in the pediatric ENT department and in the ENT Clinic of the "Sfanta Maria" Hospital, Bucharest. The patient received surgical treatment with cold instruments and CO2 and Diode LASERs vaporization, but also non-specific antiviral therapy - Isoprinosine - and specific antiviral therapy - Cidofovir.

It should be noted that the patient never needed emergency tracheostomy for acute respiratory failure.

In 2017 , the patient was diagnosed with pemphigus vulgaris that required the initiation of immunomodulatory treatment, treatment that negatively influenced the evolution of papillomatous lesions, increasing the number of local recurrences and the need for surgical intervention respectively.

The histopathological examination revealed intraepidermal blister with suprabasal acantholysis. The direct immunofluorescence showed intercellular IgG and C3 deposition in the epithelium of the pemphigus-specific oral mucosa. The detection of circulating autoantibodies against Desmoglein 3 was positive (titer 1: 320), whereas anti-Desmoglein 1 antibodies were negative.

The ENT clinical and laryngofibroscopic examinations revealed diffuse papillomatous lesions at the level of the endolarynx, especially in the vocal cord and right ventricular band, synechiae in the anterior commissure (Figure 2). Also, pemphigus-like lesions in the epiglottis, aryepiglottic folds (Figure 2) and in the gingival mucosa were revealed, and meliceric crusts at the level of the bilateral inferior nasal turbinate head.

The following were also performed: pulmonary examination (pulmonary CT scan - pulmonary cystic lesions; normal spirometry $-\mathrm{VC}=5.791(96 \%), \mathrm{FEV}_{1}=$ $4.181(83 \%), \mathrm{FEV}_{1} / \mathrm{FVC}$ ratio $=72 \%$; clinically: pulmonary vesicular breath diffusely decreased, without rales, $\mathrm{SpO} 2=96 \%$ ); normal complete blood count, coagulogram, biochemistry; normal ECG.

In the ENT\&HNS Department, "Sfanta Maria" Hospital, the patient underwent a suspension microlaryngoscopy (Figure 3) for the excision of endolaryngeal papillomatous tumor formations and intralesional injection of Cidofovir.

To date, the patient has benefited from 5 intralesional Cidofovir injection procedures. The gradual decrease in Prednisone doses $(10 \mathrm{mg} /$ day) associated with antiviral treatment allowed to reduce the number of recurrences, partial regression of papillomas and improvement of voice quality.

The peculiarity of the above case is the association of laryngeal papillomatosis with pemphigus vulgaris, situation that imposes particular difficulties in the therapeutic management of this patient. Patient with multiple surgeries during childhood, but who underwent a single surgery in the age range of $16-18$ years, 
after having been diagnosed with pemphigus vulgaris at the age of 18 , he had to undergo 8 more surgical operations. Immunomodulatory medication negatively influences the development of papillomatosis, increasing the number of local recurrences and the need for surgical intervention respectively. The treatment with intralesional Cidofovir associated with systemic corticosteroid therapy, in a low dose, allowed a reduction in the number of recurrences, partial regression of papillomas, improvement in voice quality and respiratory obstruction, decreasing the number of surgeries for the patient.
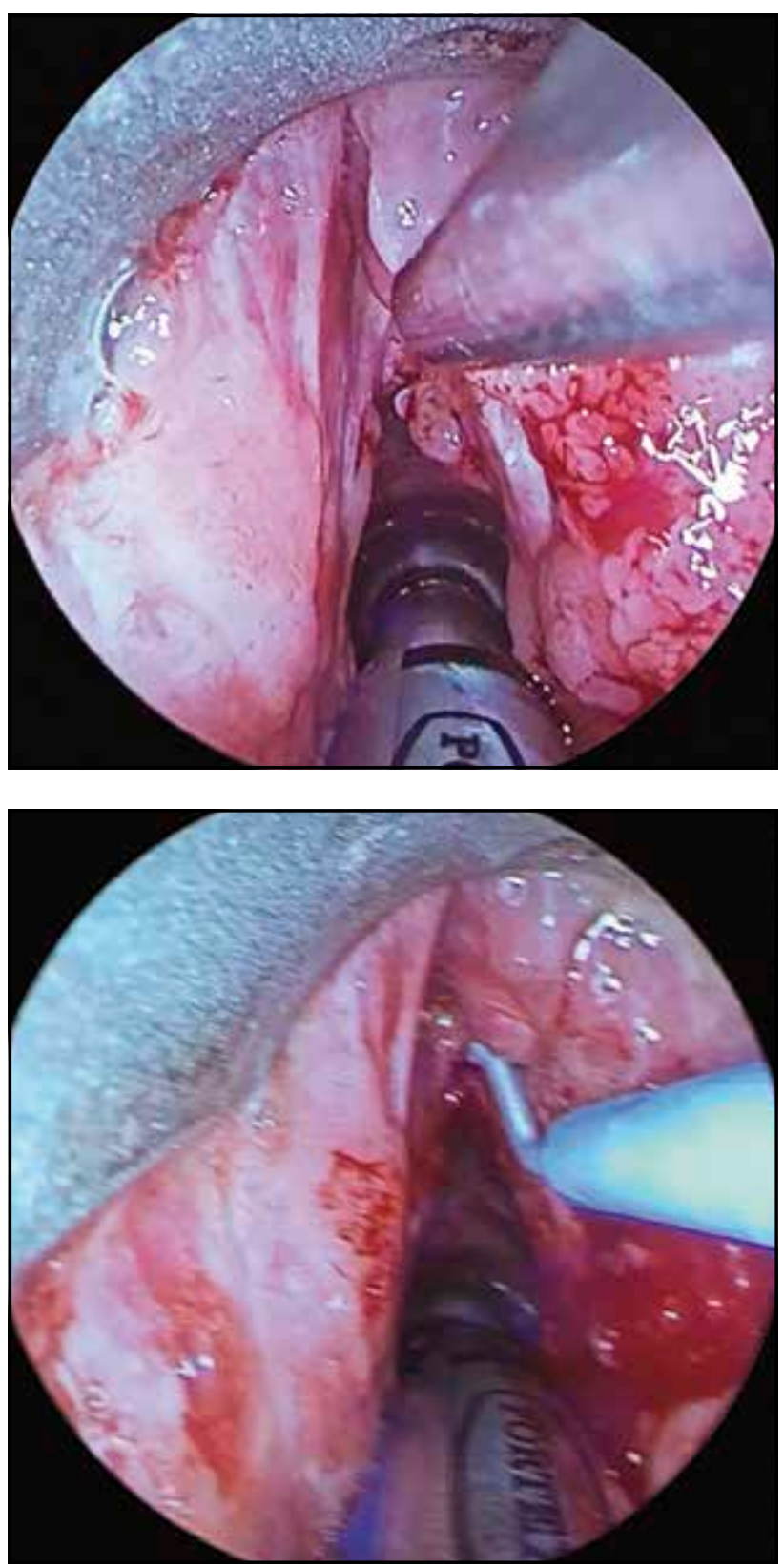

Figure 3. Intraoperative appearance - suspension laryngoscopy: papillomatous lesions in the right vocal cord and pemphigus lesions in the laryngeal vestibule are highlighted.
A therapeutic option for this patient would have been Rituximab or Bevacizumab, compounds that belong to the category of monoclonal antibodies. Rituximab is an extremely effective treatment for pemphigus, being recommended as first-line therapy, using the "protocol for rheumatoid arthritis" of 2 intravenous infusions of 1 gram, administered every 2 weeks $^{40}$. According to the reports of Heelan et al., the effect of this treatment settles slowly, in 3-6 months. Additional doses of rituximab may be recommended to prevent relapses $(500 \mathrm{mg}$ intravenous infusions every 6-12 months ${ }^{40}$ ). Specialized studies highlight the efficacy of monoclonal antibodies on HPV infection ${ }^{41,42}$.

Another option would be to use methotrexate weekly, as an adjuvant to systemic steroids. The advantages of using methotrexate are that it is not expensive and has a low risk of worsening HPV infection.

Another option may be the use of intravenous immunoglobulins, as they have a very low risk of worsening HPV infection.

\section{CONCLUSIONS}

The association of recurrent juvenile respiratory papillomatosis with pemphigus vulgaris is not very common. The evolution of the patient is unknown even with treatment.

Immunosuppressive medication negatively influences the development of papillomatosis, increasing the number of local recurrences and the need for surgical intervention respectively, due to increased viral multiplication and proliferation of papillomatous lesions. In our opinion, these 2 disorders act for each other as a negative morbidity factor.

Further research that will identify the pathophysiological mechanisms and the curative treatment will positively influence the evolution and prognosis of these patients.

Contribution of authors: All contributors meet the criteria for authorship.

Conflict of interest: The authors have no conflict of interest.

Acknowledgements: None

\section{REFERENCES}

1. Gearhart PA, Randall TC, Buckley RM, Higgins RV. Human Papillomavirus (HPV). [Internet]. Medscape [updated Feb 20, 2020]. Available from: https://emedicine.medscape.com/article/219110-overview.

2. Sarafoleanu C. Tumorile benigne ale laringelui. In: Popescu I, Ciuce C (eds); Sarafoleanu C (coord). Tratat de chirurgie. Editia a II-a. Vol I.: Otorinolaringologie si chirurgie cervico-faciala. Editura Academiei Romane, Bucuresti; 2012, p.199-217.

3. Gheorghe DC. Patologia laringiana pediatrica. In: Popescu I, Ciuce C (eds); 
Sarafoleanu C (coord). Tratat de chirurgie. Editia a II-a. Vol I.: Otorinolaringologie si chirurgie cervico-faciala. Editura Academiei Romane, Bucuresti; 2012, p.568-601.

4. McCane DJ. Papillomaviruses. In: Zuckermann AJ, Banatvala JE, Pattison JR (eds). Principles and practice of clinical virology. Fourth Edition. John Wiley \& Sons Ltd; 2000, p.607-18. DOI: 10.1002/0470842474.ch22.

5. de Villiers EM, Fauquet C, Broker TR, Bernard HU, zur Hausen H. Classification of papillomaviruses. Virology. 2004;324(1):17-27.

6. Derkay CS. Task force on recurrent respiratory papillomas. A preliminary report. Arch Otolaryngol Head Neck Surg. 1995;121(12):1386-91. DOI: 10.1001/archotol.1995.

7. Chow VT, Leong PWF. Complete nucleotide sequence, genomic organization and phylogenetic analysis of a novel genital human papillomavirus type, HLT7474-S. J Gen Virol. 1999;80(Pt 11):2923-9. DOI: 10.1099/0022-1317-8011-2923.

8. Farmer AD, Calef CE, Millman K, Myers GL. The human papillomavirus database. J Biomed Sci. 1995;2(2):90-104.

9. Muñoz N, Bosch FX, de Sanjosé S, Herrero R, Castellsagué X, Shah KV, et al. Epidemiologic classification of human papillomavirus types associated with cervical cancer. N Engl J Med. 2003;348(6):518-27.

10. Doorbar J. Molecular biology of human papillomavirus infection and cervical cancer. Clin Sci (Lond). 2006;110(5):525-41.

11. Hebner CM, Laimins LA. Human papillomaviruses: basic mechanisms of pathogenesis and oncogenicity. Rev Med Virol. 2006;16(2):83-97.

12. Durst M, Kleinheinz A, Hotz M, Gissmann L. The physical state of human papillomavirus type 16 DNA in benign and malignant genital tumours. J Gen Virol. 1985;66(Pt 7):1515-22.

13. Lehoux M, D'Abramo CM, Archambault J. Molecular mechanisms of human papillomavirus-induced caricnogenesis. Public Health Genomics. 2009;12(56):268-80. DOI: 10.1159/000214918.

14. Hoory T, Monie A, Gravitt P, Wu TC. Molecular epidemiology of human papillomavirus. J Formos Med Assoc. 2008;107(3):198-217. DOI: 10.1016/ S0929-6646(08)60138-2.

15. Egawa K. Do human papillomaviruses target epidermal stem cells? Dermatology. 2003;207(3):251-4. DOI: 10.1159/000073085.

16. Gravitt PE. Evidence and impact of human papillomavirus latency. Open Virol J. 2012;6:198-203. DOI: 10.2174/1874357901206010198.

17. Egawa N, Egawa K, Griffin H, Doorbar J. Human papillomaviruses; epithelial tropism, and the development of neoplasia. Viruses. 2015;7(7):386-90. DOI: 10.3390/v7072802.

18. Bonagura VR, Vambutas A, DeVoti JA, Rosenthal DW, Steinberg BM, Abramson AL, et al. HLA alleles, IFN-gamma responses to HPV-11 E6, and disease severity in patients with recurrent respiratory papillomatosis. Hum Immunol. 2004;65(8):773-82. DOI: 10.1016/j.humimm.2004.05.014.

19. Gelder CM, Williams OM, Hart KW, Wall S, Williams G, Ingrams D, et al. HLA class II polymorphism amd susceptibility to recurrent respiratory papillomatosis. J Virol. 2003;77(3):1927-39. DOI: 10.1128/JVI.77.3.19271939.2003.

20. Snowden RT, Thomson J, Horwitz E, Stocks RM. The prediction value of serum interleukins in recurrent respiratory papillomatosis: a preliminary study. Laryngoscope. 2001;111(3):404-8.

21. Williams OM, Hart KW, Wang ECY, Geller CM. Analysis of CD4+ T-cell responses to human papillomavirus (HPV) type $11 \mathrm{~L} 1$ in healthy adults reveals a high degree of responsiveness and cross-reactivity with other HPV types. J Virol. 2002;76(15):7418-29. DOI: 10.1128/JVI.76.15.7418-7429.2002.

22. Hatam LJ, DeVoti JA, Rosenthal DW, Lam F, Abramson AL, Steinberg BM, et al. Immune suppression in premalignant respiratory papillomas: enriched functional CD4+Foxp3+ regulatory T cells and PD-1/PD-L1/L2 expression. Clin Cancer Res. 2012;18(7):1925-35.
23. Ahn J, Bishop JA, Roden RBS, Allen CT, Best SRA. The PD-1and PD-L1 pathway in recurrent respiratory papillomatosis. Laryngoscope. 2018;128(1):E2732. DOI: 10.1002/lary.26847.

24. Escobedo AR, Santillana KMS, Gonzalez JLT. Recurrent respiratory papillomatosis: review and treatment update. J Otolaryngol Rhinol. 2018;4:040. DOI: $10.23937 / 2572-4193.1510040$.

25. Derkay CS, Hester RP, Burke B, Carron J, Lawson L. Analysis of a staging assessment system for prediction of surgical interval in recurrent respiratory papillomatosis. Int J Pediatr Otorhinolaryngol. 2004;68(12):1493-8.

26. Ivancic R, Iqbal H, deSilva B, Pan Q, Matrka L. Current and future management of recurrent respiratory papillomatosis. Laryngoscope Investig Otolaryngol. 2018;3(1):22-34. DOI: 10.1002/lio2.132.

27. Bonagura VR, Hatam LJ, Rosenthal DW, de Voti JA, Lam F, Steinberg BM, et al. Recurrent respiratory papillomatosis: a complex defect in immune responsiveness to human papillomavirus-6 and -11. APMIS. 2010;118(67):455-70. DOI: 10.1111/j.1600-0463.2010.02617.x.

28. DeVoti J, Hatam L, Lucs A, Afzal A, Abramson A, Steinberg B, et al. Decreased Langerhans cell responses to IL-36gamma: altered innate immunity in patients with recurrent respiratory papillomatosis. Mol Med. 2014;20:37280. DOI: 10.2119/molmed.2014.00098.

29. Rhea WG Jr, Bourgeois BM, Sewell DR. Condyloma acuminata: a fatal disease? Am Surg. 1998;64(11):1082-7.

30. Eaton WW, Rose NR, Kalaydjian A, Pedersen MG, Mortensen PB. Epidemiology of autoimmune diseases in Denmark. J Autoimmun. 2007;29(1):1-9.

31. Cooper GS, Bynum ML, Somers EC. Recent insights in the epidemiology of autoimmune diseases: Improved prevalence estimates and understanding of clustering of diseases. J Autoimmun. 2009;33(3-4):197-207. DOI: 10.1016/j. jaut.2009.09.008.

32. Chandrashekara S. The treatment strategies of autoimmune disease may need a different approach from conventional protocol: a review. Indian J Pharmacol. 2012;44(6):665-71. DOI: 10.4103/0253-7613.103235.

33. Rosenblum MD, Gratz IK, Paw JS, Abbas AK. Treating human autoimmunity: current practice and future prospects. Sci Transl Med. 2012;4(125):125sr1. DOI: 10.1126/scitranslmed.3003504.

34. Hall BM. Corticosteroids in autoimmune diseases. Aust Prescr. 1999;22:9-11. DOI: 10.18773/austprescr.1999.008.

35. Johnson PJ, McFarlane IG, Williams R. Azathioprine for long-term maintenance of remission in autoimmune hepatitis. $\mathrm{N}$ Enlg $\mathrm{J}$ Med. 1995;333(15):958-63.

36. de Winter BCM, van Gelder T. Therapeutic drug monitoring for mycophenolic acid in patients with autoimmune disease. Nephrol Dial Transplant. 2008;23(11):3386-8. DOI: 10.1093/ndt/gfn497.

37. Zeina B, Sakka N, Mansoor S. Pemphigus vulgaris. [Internet]. Medscape [updated Jun 14,2018]. Available online: https://emedicine.medscape.com/ article/1064187-overview\#a6.

38. Fitzpatrick RE, Newcomer VD. The correlation of disease activity and antibody titers in pemphigus. Arch Dermatol. 1980;116(3):285-90.

39. Lombardi ML, Mercuro O, Ruocco V, Lo Schiavo A, Lombari V, Guerrera V, et al. Common human leukocyte antigen alleles in pemphigus vulgaris and pemphigus foliaceus Italian patients. J Invest Dermatol. 1999;113(1):107-10.

40. Heelan K, Al-Mohammedi F, Smith MJ, Knowles S, Lansang P, Walsh S, et al. Durable remission of pemphigus with fixed-dose rituximab protocol. JAMA Dermatol. 2014;150(7):703-8. DOI: 10.1001/jamadermatol.2013.6739.

41. Wang Y, Shang Q, Xu W, Di L, Gu H, Wei L. Characterization of two new monoclonal antibodies against human papillomavirus type $16 \mathrm{~L} 1$ protein. Diagn Pathol. 2014;9:101. DOI: 10.1186/1746-1596-9-101.

42. Mohr M, Schliemann C, Biermann C, Schmidt LH, Kessler T, Schmidt J, et al. Rapid response to systemic bevacizumab therapy in recurrent respiratory papillomatosis. Oncol Lett. 2014;8(5):1912-8. DOI: 10.3892/ol.2014.2486. 